NOTA CIENTÍFICA

\section{Hábitos alimentarios de infantiles de Pleurodema nebulosum (Anura: Leptodactylidae), en Matagusanos, San Juan, Argentina Alimentary habits of infantile Pleurodema nebulosum (Anura: Leptodactylidae), in Matagusanos, San Juan, Argentina}

\author{
Eduardo Sanabria, Lorena Quiroga y Juan Carlos Acosta
}

Departamento de Biología e Instituto y Museo de Ciencias Naturales, Facultad de Ciencias Exactas Físicas y Naturales, Universidad Nacional de San Juan, Avenida España 400 (Norte). Sta. Maria de Oro 367(s), CP: 5400, San Juan, Argentina

Email Eduardo Sanabria: sanabria_ea@yahoo.com.ar Email Lorena Quiroga: quiroga_lore@yahoo.com.ar Email Juan Carlos Acosta: jcacosta@sinectis.com.ar

Presentado: $\quad$ 29/08/2006 Aceptado: $\quad 22 / 04 / 2007$

\section{Resumen}

Los hábitos alimentarios de Pleurodema nebulosum en el oeste de Argentina son desconocidos. El objetivo de este trabajo es analizar la dieta de una población de infantiles de $P$. nebulosum. Se analizaron 34 estómagos, se clasificaron taxonómicamente sus presas y se midieron (ancho y largo), se calculó su volumen con la fórmula de la esfera elipsoide, se estimó además la frecuencia de ocurrencia y abundancia. Los IRI más importantes fueron: Hemiptera (1867), Hymenoptera (hormigas) (1431), Coleoptera (127), entre otros. Las presas que presentaron mayor porcentaje de frecuencia fueron los Hymenoptera $(48,5 \%)$, Hemiptera $(26,9 \%)$ y Coleoptera $(10,8 \%)$. El valor de diversidad media fue de $0.19(S D=0,2)$ y la amplitud del nicho trófico fue de 3,17 . Este valor indicaría una posible especialización y considerando la baja diversidad encontrada, podría estar reflejando la escasa disponibilidad de presas en ese momento.

Palabras claves: Argentina, dieta, infantiles, Pleurodema nebulosum, San Juan.

\section{Abstract}

The alimentary habits of Pleurodema nebulosum in the west of Argentina are unknown. The objective in this work was to analyze the diet of a population of infantile of $P$. nebulosum. We analyze 34 stomachs, the preys were classified and measured (wide and long), and their volumes were estimated with the sphere ellipsoid. It was considered the occurrence frequency and the abundance. The IRI most important were: Hemiptera (1867), Hymenoptera (1431), Coleoptera (127), among others. The preys with highest presence were the Hymenoptera $(48,5 \%)$, Hemiptera $(26,9 \%)$ y Coleoptera $(10,8 \%)$. The value of mean diversity was of $0,19(S D=0,2)$ and the width of trophic niche was of 3,17 ; it could be indicate a possible specialization. But, in considering the low diversity values, it could be for the scarce readiness of preys in that moment too.

Keywords: Argentina, diet, infantile, Pleurodema nebulosum, San Juan.
Pleurodema nebulosum es un típico habitante de zonas áridas del oeste de Argentina, desde Catamarca hasta Río Negro. La distribución de esta especie coincide con la provincia fitogeográfica del Monte (Cei, 1980). La alimentación es considerada, junto con el tiempo y lugar de actividad, una de las principales dimensiones del nicho ecológico de cualquier especie animal (Pianka, 1973; Schoener, 1974). Las estrategias alimentarias incluyen el número de presas y su ubicación, como también su captura e ingestión. La selección de las presas esta influenciada tanto por el tipo de hábitat, como por la estacionalidad, de acuerdo a esto los ítems alimentarios pueden variar en cantidad y calidad (Dullman y Trueb, 1986).

El objetivo de la presente nota es analizar la dieta de una población de infantiles de $P$. nebulosum en el barreal de Matagusanos, el cual se encuentra ubicado a $40 \mathrm{Km}$ al norte de la Ciudad de San Juan sobre la Ruta Nacional n. 040.

El barreal de Matagusanos pertenece a la provincia Fitogeográfica del Monte (Cabrera, 1994) a 800 m de altitud, en una región de clima árido con una temperatura media anual de $20{ }^{\circ} \mathrm{C}$ y una máxima media anual de $40{ }^{\circ} \mathrm{C}$ y mínima media anual de $16^{\circ} \mathrm{C}$, con las lluvias concentradas en verano con un promedio anual de $84 \mathrm{~mm}$ (Poblete y Mineti, 1999).

Manualmente fueron recolectados 34 individuos el día 21 de abril de 2005 , entre las 15 y $18 \mathrm{~h}$. Los individuos fueron fijados en formaldehído al 10\% y luego conservados en alcohol al $70 \%$.
En cada individuo se tomó el Largo hocico cloaca (LHC), ancho de la boca a la altura de las comisuras (AB) y peso de los mismos con una balanza de $0,1 \mathrm{~g}$ de precisión.

El contenido de los estómagos fue analizado, determinándose las presas taxonómicamente al nivel de orden y familia. Cada presa fue medida en su largo (L) y ancho (A) con el fin de calcular su volumen con la fórmula de una esfera elipsoide (Dunham, 1983).

Para determinar la contribución de cada categoría de alimento a la dieta se aplicó el Índice de Importancia Relativa (IRI) propuesto por Pinkas et al. (1971). Para calcular la jerarquización de la dieta se aplicó al valor de IRI el índice de jerarquización (DJ), que toma el valor más alto del IRI y calcula el porcentaje de todos los demás valores a partir de él. Si el porcentaje del ítem presa se encuentra incluido entre el 100 y $75 \%$ se lo considera fundamental; si se ubica entre el 75 y $50 \%$ se lo considera secundario; si el valor se ubica entre 50 y $25 \%$ es accesorio, y si está por debajo del $25 \%$ se lo considera accidental (Martori, 1991). La amplitud del nicho trófico fue calculada a partir de la utilización del índice de Levins (1968). Además se calculó la diversidad trófica $(H)$ según el criterio de Hurtubia (1973).

Los datos obtenidos fueron analizados mediante estadística descriptiva y no paramétrica.

Del total de los individuos capturados $(n=34)$ solo cinco no presentaron contenido estomacal. Los 29 individuos analizados, 
Tabla 1. Lista taxonómica del contenido estomacal, $\mathrm{N}=$ número total de ítems de los 29 contenidos estomacales; \%N= porcentaje de la categoría en el total de las presas; \%V= porcentaje del volumen de la categoría en el volumen total; \%FO= porcentaje de la frecuencia de ocurrencia; IRI= Índice de importancia relativa de cada categoría. Se señalan en negrita los Ítems presa (OTU) con sus respectivos IRI. En la columna DJ se visualizan los porcentajes del índice de jerarquización de las presas.

\begin{tabular}{|c|c|c|c|c|c|c|c|}
\hline & & $\mathbf{N}$ & $\% \mathbf{N}$ & $\% \mathrm{~V}$ & $\%$ FO & I.R.I. & DJ \\
\hline \multirow[t]{2}{*}{ Hymenopte } & $\stackrel{a}{\text { Formicidae }}$ & 101 & 48,5 & 9,7 & 24,6 & $\begin{array}{l}\mathbf{1 4 3 8} \\
1431\end{array}$ & $77 \%$ \\
\hline & Vespidae & 2 & 0,96 & 2,16 & 2,7 & 8,4 & \\
\hline Coleoptera & $\begin{array}{l}\text { Carabidae } \\
\text { Escarabeidae } \\
\text { Estafilinidae } \\
\text { Coccinelidae } \\
\text { Cerambicidae } \\
\text { Larvas }\end{array}$ & $\begin{array}{l}6 \\
1 \\
7 \\
3 \\
1 \\
5\end{array}$ & $\begin{array}{c}2,8 \\
0,48 \\
3,3 \\
1,4 \\
0,48 \\
2,4\end{array}$ & $\begin{array}{c}3,6 \\
0,19 \\
0,67 \\
9,4 \\
0,04 \\
2,3\end{array}$ & $\begin{array}{l}5,4 \\
1,3 \\
5,4 \\
4,1 \\
1,3 \\
5,4\end{array}$ & $\begin{array}{c}\mathbf{1 2 b} \\
34 \\
0,87 \\
21,4 \\
44,2 \\
0,67 \\
25,3\end{array}$ & $6,1 \%$ \\
\hline \multirow{2}{*}{\multicolumn{2}{|c|}{$\begin{array}{l}\text { Isoptera } \\
\text { Diptera }\end{array}$}} & 11 & 5,2 & 0,45 & 12,3 & 69,5 & $3,7 \%$ \\
\hline & & 8 & 3,8 & 1 & 5,4 & 25,9 & $1,3 \%$ \\
\hline \multicolumn{2}{|c|}{ Hemiptera } & 56 & 26,9 & 64,2 & 20.5 & 1867 & $100 \%$ \\
\hline \multicolumn{2}{|c|}{ Lepidoptera (Larva) } & 6 & 2,8 & 3,2 & 3,8 & 23 & $1,2 \%$ \\
\hline \multirow{2}{*}{\multicolumn{2}{|c|}{$\begin{array}{l}\text { Aranae } \\
\text { Total }\end{array}$}} & 1 & 0,48 & 0,27 & 1,3 & 0,97 & $0,05 \%$ \\
\hline & & 1470 & 100 & 100 & & & \\
\hline
\end{tabular}

tuvieron un LHC medio de 17,8 $\mathrm{mm}(\mathrm{SD}=2,5)$, el ancho de la boca medio fue de $8,1 \mathrm{~mm}(\mathrm{SD}=1,3)$ y un peso promedio de $1,2 \mathrm{~g}(\mathrm{SD}=0,8)$. Se identificaron un total de 208 presas, con un promedio de número de presas por estómago de $7,9(\mathrm{SD}=8,1)$, contenidas en 13 categorías taxonómicas todas correspondientes a la fracción animal (Tabla1).

Con la agrupación de éstas siguiendo el criterio de unidad taxonómica operacional (OTU) (Sneath y Sokal, 1973) se consideraron 10 ítems presa: Hymenoptera, Coleoptera, Isopoda, Diptera, Heminoptera, Lepidoptera, Aranae. Según el índice de jerarquización agrupamos en: Ítems fundamentales para la dieta: Heminoptera (100\%), Hymenoptera (77\%); Ítems acidentales de la dieta: Coleoptera (6,7\%), Isopoda (3,7\%), Diptera (1,3\%), Lepidoptera (1,2\%), Aranae (0,05\%).

La contribución de cada categoría se ve reflejada por el índice de importancia relativa (IRI) el cual presenta el mayor valor con Hemiptera (1867), Hymenoptera (hormigas) (1431), Coleoptera (127), Isoptera (69), Diptera (26), Lepidoptera larvas $(23)$ y Otros $(9,37)$ donde se agruparon los valores de los ítems alimentarios de más bajo aporte a la dieta. Las presas que presentaron mayor porcentaje de frecuencia fueron los Hymenoptera $(48,5 \%)$ y le siguen en orden de importancia Hemiptera $(26,9 \%)$ y Coleoptera (10,8\%). A diferencia de lo encontrado por Sanabria et al., (resultados no publicados) para los adultos de esta especie donde el ítem alimentario más importante fue Coleoptera, conservándose como ítem con mayor frecuencia a Hymenoptera.

El valor de diversidad media fue de $0,19(\mathrm{SD}=0,2)$, la curva de novedades tróficas acumuladas se estabilizo en el estómago número 21 indicando la muestra mínima para este estudio. La amplitud del nicho trófico $(\mathrm{Nb})$ fue de 3,17. Este valor indicaría una posible tendencia hacia la especialización y considerando la baja diversidad encontrada, podría estar reflejando la escasa disponibilidad en la diversidad de presas en ese momento. La amplitud del nicho de los adultos $(\mathrm{Nb}=2,9)$ para el mes de enero en el mismo lugar también indica una tendencia a la especialización en la captura del alimento (Sanabria et al., resultados no publicados). Por otro lado es necesario resaltar la particularidad del ambiente considerado ("barreal") y la escasa cobertura vegetal y su influencia en la diversidad de artrópodos. Respecto a la relación predador presa no se encontramos relaciones (Spearman) significativas entre el LHC y el largo medio de las presas (p>0,22), el LHC y el ancho medio de la presa $(\mathrm{p}>0,89)$ y el LHC y el volumen medio de presas por estómago $(p>0,65)$. Los individuos se encuentran en un rango entre 14 y $22 \mathrm{~mm}$ de LHC no se observa una selección de tamaño de presa en función del tamańo del predador. Esto es coincidente con lo hallado en Chile para Pleurodema thaul donde el número de presas, como el tamaño de éstas, no tiene relación alguna con el tamańo del predador (Diaz-Páez et al., 2003). No se encontraron correlaciones (Spearman) entre el ancho de la boca y el Largo medio de presa ( $p>0,57)$, ancho de la boca y ancho medio de presa $(\mathrm{p}>0,81)$ y ancho de la boca y volumen medio de las presas por estómago $(\mathrm{p}>0,96)$.

\section{Literatura citada}

Cabrera A. L. 1994. Enciclopedia Argentina de Agricultura y Jardinería, Tomo II, Regiones Fitogeográficas Argentinas, Editorial ACME S.A.C.I.Pp. 36-42.

Cei J. M. 1980. Amphibians of Argentina. Monitore Zoologico Italiano, Italian Journal of Zoology, Monografia 2: 1-609.

Duellman W. E. \& L. Trueb. 1986. Biology of Amphibians. MacGraw-Hill Book Company. Pp. 613.

Gallardo J. M. 1987. Anfibios argentinos, Guía para su Identificación. Biblioteca Mosaico. Librería agropecuaria S.A. 1ra. Edición. Pp. 98.

Lavilla E.O. \& M. Rouges. 1992. Reproducción y Desarrollo de Anuros Argentinos. Asociación Herpetológica Argentina serie de Divulgación n. ${ }^{\circ}$ 5. Pp. 66.

Martori A. 1991. Alimentación de los adultos de Eoproctus asper (Dugés 1853) en la montaña media del Pirineo catalán. (España). Rev. Esp. Herp. 5: 23-36.

Dias-Paez H. \& J.C. Ortiz. 2003. Habitos alimentarios de Pleurodema thaul (Anura: Leptodactylidae), en Concepción, Chile. Gayana 67 (1): 25-32.

Perotti M.G. 1997. Modos Reproductivos y variables reproductivas cuantitativas de un ensamble de anuros del Chaco semiárido, Salta Argentina. Revista Chilena de Historia Natural 70: 277-288.

Pianka E. R. 1973. The structure of lizard communities. Ann. Rev. Ecol. Syst. 4: 53-74.

Poblete, A. \& Minetti, J., 1999. Configuración espacial del Clima de San Juan. Síntesis del Cuaternario de la Provincia de San Juan. $11^{\circ}$ Reunión de Campo del Cuaternario. CD-ROM. INGEO Universidad Nacional de San Juan. San Juan, Argentina.

Schoener, T. W. 1974. Resource partitioning in ecological communities. Science. 189: 27-39. 